\title{
GOSSIP: a vertex detector combining a thin gas layer as signal generator with a CMOS readout pixel array
}

\author{
M. Campbell ${ }^{\mathrm{a}}$, E.H.M. Heijne ${ }^{\mathrm{a}}$, X. Llopart $^{\mathrm{a}}$, P. Colas $^{\mathrm{b}}$, A. Giganon $^{\mathrm{b}}$, Y. Giomataris ${ }^{\mathrm{b}}$, M. Chefdeville $^{\mathrm{c}}$,

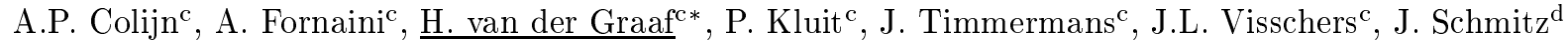 \\ ${ }^{\text {a } C E R N / M e d i P i x ~ C o n s o r t i u m, ~ G e n e v a, ~ S w i t z e r l a n d ~}$ \\ ${ }^{\text {b} D A P N I A, ~ C E A ~ S a c l a y, ~} 91191$ Gif sur Yvette Cedex, France \\ c NIKHEF, Amsterdam, The Netherlands \\ ${ }^{\mathrm{d}}$ Univ. of Twente/MESA, The Netherlands
}

A small TPC has been read out by means of a Medipix 2 chip as direct anode. A Micromegas foil was placed $50 \mu \mathrm{m}$ above the chip, and electron multiplication occurred in the gap. With a He/Isobutane 80/20 mixture, gas multiplication factors up to tens of thousands were achieved, resulting in an efficiency for detecting single electrons of better than $90 \%$. We recorded many image frames containing 2D images with tracks from cosmic muons. Along these tracks, electron clusters were observed, as well as $\delta$-rays. With a gas layer thickness of only one $\mathrm{mm}$, the device could be applied as vertex detector, outperforming all Si-based detectors.

\section{Introduction}

Recently, we presented first results on the readout of gas filled drift chambers by means of a pixel segmented active anode [1] [2]. Images of the interaction of ${ }^{55} \mathrm{Fe}$ quanta with the gas were obtained. This is shown in Figure 1 for data taken at a rather low gas amplification factor of around $1 \mathrm{k}$. Both a 3-layer GEM structure in a $10 \mathrm{~cm}$ drift length detector and a single-layer Micromegas foil in a $15 \mathrm{~mm}$ drift length chamber were used. In the first setup, rather diffuse blobs of hit pixels were seen. This was due to the transverse diffusion of the drifting electrons in the gas and, possibly, a defocusing effect on the electron avalanches at the exit of the GEM holes. As expected, the images using the Micromegas setup were much sharper, because a) of the reduced maximum drift length, b) the Micromegas foil is at a distance of only $50 \mu \mathrm{m}$ from the anode. To prove the feasibility of the detection of single drifting electrons from minimum ionising particles, the same Micromegas/pixel sensor structure was used operating at gas multiplication factors of up to $20 \mathrm{k}$.

\footnotetext{
*Corresponding author; e-mail: vdgraaf@nikhef.nl
}

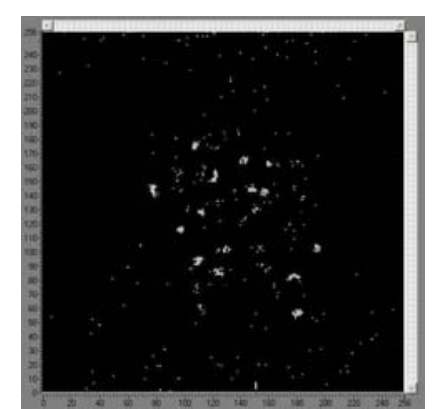

Figure 1. Image acquired with the Medipix2/Micromegas prototype TPC.

\section{Experimental Setup}

The layout of the test chamber is shown in Figure 2. A cathode foil is fixed above an aluminum base plate by means of spacers, forming a drift gap of $15 \mathrm{~mm}$. The Medipix 2 readout chip is mounted on a brass pedestal and placed flush with the base plate upper plane. On top of the chip a Micromegas foil, fixed on a frame, is held in position by means of two silicon rubber strings 


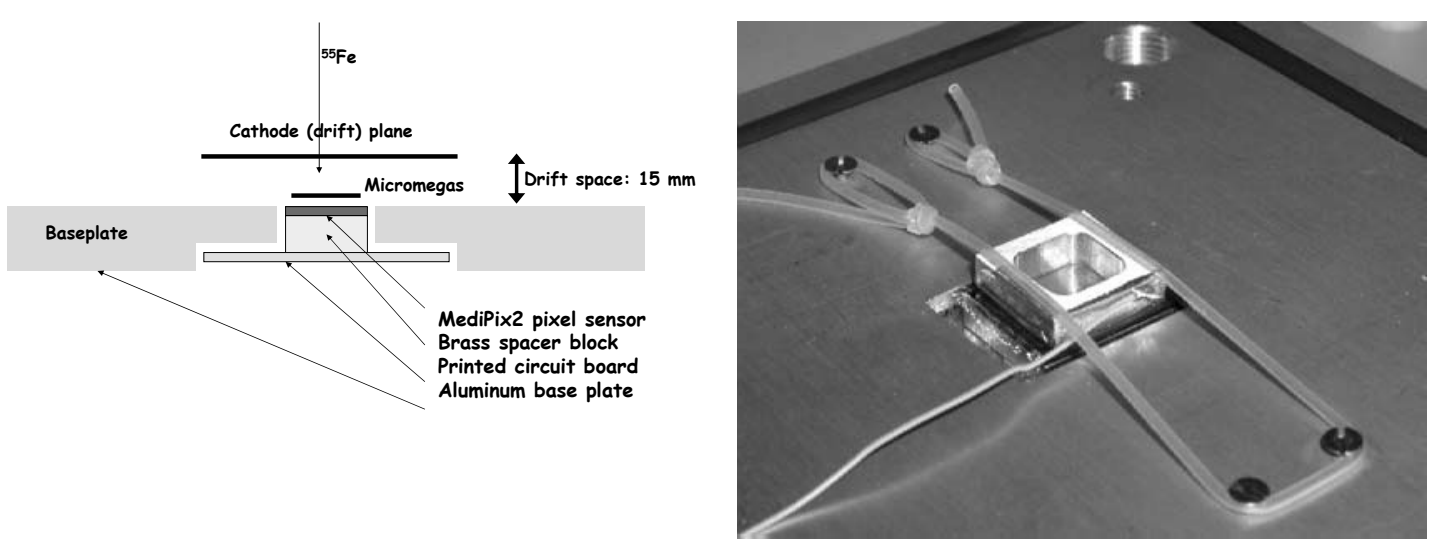

Figure 2. Left: The layout of the chamber with the Medipix2, the Micromegas and the drift gap; Right: The mounting of the Micromegas onto the Medipix2 sensor.

(see Figure 2).

A Medipix2 chip [3] [4] [5] was applied as experimental readout device. This CMOS chip contains a square matrix of $256 \times 256$ pixels, with a pixel size of $55 \times 55 \mu^{2}$. Each pixel is equipped with a low-noise preamp, discriminator, threshold DACs, a 14-bit counter and communication logic. One edge of the chip has aluminum bonding pads. The outer dimensions of the chip are $16.12 \times$ $14.11 \mathrm{~mm}^{2}$. The Medipix2 chip has been designed for X-ray imaging applications. For that particular application, an X-ray semiconductor converter (i.e. Si or CdZnTe), in the form of a corresponding pixel matrix, is mounted onto the Medipix2 chip, by means of bump-bonding. In our application we use the "naked" Medipix2 chip, without an X-ray convertor. For each pixel the number of absorbed X-ray quanta in a given acquisition time is counted, and the combined pixel content forms the X-ray image.

Originally, each pixel of the Medipix2 chip is covered for a large part with an insulating passivation layer; the conductive pad (octagonally shaped, $25 \mu \mathrm{m}$ wide) is large enough to accommodate a bump bond sphere. In order to prevent discharges, the Medipix2 wafers were post-processed by MESA+ [6]. The post-processing consisted of a deposition of a thin aluminum layer using liftoff lithography. This allows deposition of metal on the anode matrix without modification of the bond pads. The pixel pads were thus enlarged to reach a metal coverage of $80 \%$ of the anode plane. Electrical tests showed that the preamplifier functionality was unaffected by this post-processing.

The Micromegas [7] is a copper foil, thickness $5 \mu \mathrm{m}$, with holes of $35 \mu \mathrm{m}$ diameter in a square pattern with $60 \mu \mathrm{m}$ pitch [8]. At the foil side facing the Medipix2 chip, poly-imide pillars (height $50 \mu \mathrm{m}$, diameter $80-140 \mu \mathrm{m}$, pitch (square) 840 $\mu \mathrm{m})$ are attached. When the voltage on the Micromegas was applied $(200-500 \mathrm{~V})$, the electrostatic force pulls the mesh towards the chip, and the insulating pillars define the proper gap size. The Micromegas, in its frame, was held on the Medipix2 chip by means of two silicon rubber strings.

\section{Results}

The Medipix2 sensor can be externally enabled and stopped, followed by a readout sequence in which the pixel counter data is transfered to a computer. We enabled the counters during an exposure time of 15 or $60 \mathrm{~s}$, followed by recording the image frame in the form of $65 \mathrm{k}$ counter contents. No trigger was applied.

The Medipix chip, acting as direct anode, was at ground potential. With a He/Isobutane $80 / 20$ 

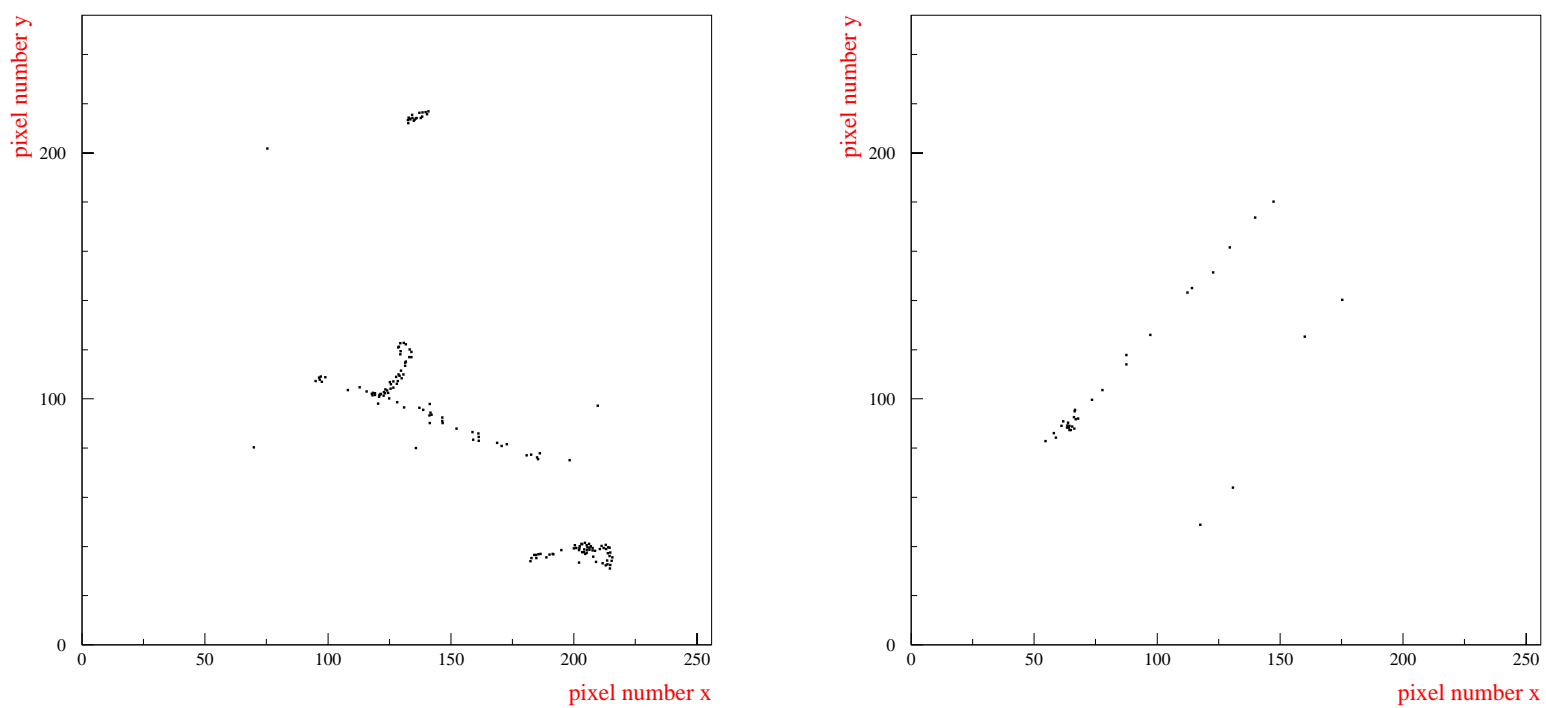

Figure 3. Left: Image recorded from the Medipix2/Micromegas prototype TPC showing a cosmic charged particle track together with a $\delta$-electron; Right: Image recorded from the Medipix2/Micromegas prototype TPC showing selected cosmics charged particle tracks.

mixture, we observed signals from ${ }^{55} \mathrm{Fe}$ events with $-390 \mathrm{~V}$ on the Micromegas and $-1000 \mathrm{~V}$ on the drift cathode plane. This is expected given the large density of primary electrons in the interaction point [1] and the gain at this voltage of about $1 \mathrm{k}$. We then increased the voltage on the Micromegas to $-470 \mathrm{~V}$, corresponding to a gain of approximately $19 \mathrm{k}$. With a threshold setting of $3000 e^{-}$, we expect a single electron efficiency of $85 \%$, and indeed cosmic rays were observed.

Some typical events are shown in Figure 3 . The left plot shows a cosmic muon that knocks out a delta electron. The right plot shows a selected cosmic muon. In this event the effect of diffusion can be observed in the spread of the hits along the track.

\section{The integration of the gas gain grid}

At the University of Twente, a 'wafer post process' is being developed by which a gas gain grid is built onto a full wafer containing pixel chips, forming a monolithic readout unit. In its simplest form, the grid takes the form of a Micromegas. First experiments to manufacture 'integrated grids' (InGrids) are shown in Figure 4.

\section{GOSSIP (Gas On Slimmed SIlicon Pix- els)}

Following the same principle, one can now also envisage a thin gas detector, consisting of a gas layer of only $1 \mathrm{~mm}$ thickness and read out by a CMOS pixel array on a thinned silicon substrate. Such a detector is fast and could be used as a vertex detector at the SuperLHC (the luminosity upgrade of the LHC), outperforming all Si-based vertex detectors.

\subsection{The performance of GOSSIP}

In the following we anticipate on the reachable performance of such a detector.

Ageing: Although the gas will be flushed, wellknown chamber ageing processes may take place, depositing matter on the anode plane, InGrid or the cathode plane. There are three reasons why 

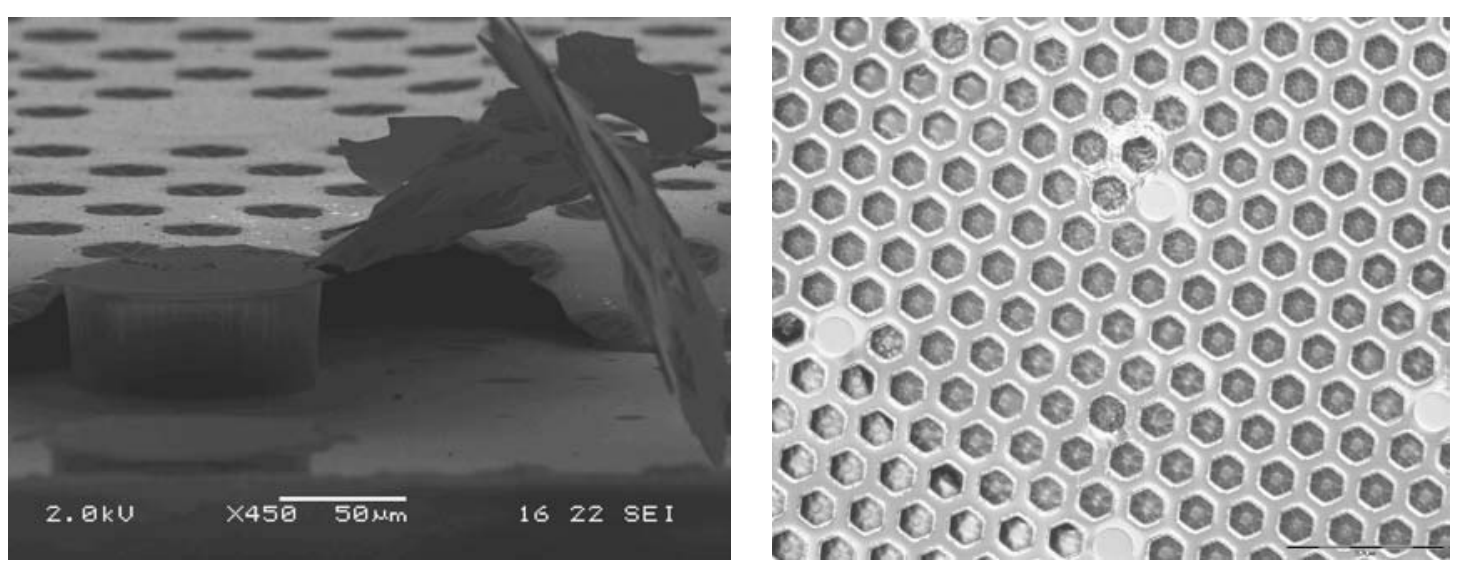

Figure 4. Two views of first trials for the production of 'integrated grids' (InGrids). The left-hand view is a side 'cut' view.

we expect GOSSIP to survive a high radiation dose:

- Due to the low input pad capacity, a low gas gain can be applied;

- The ratio of the gas volume and the total anode pad surface is very small compared to the corresponding ratio of a wire chamber, in which all avalanches end onto the surface of a thin wire;

- The electric field at a (flat) anode pad is a factor three lower than the field at a wire surface. In an avalanche, the electron energy is proportionally small and the probability to create chemical radicals is smaller than in wire chambers.

Several authors have indicated that indeed ageing effects, in GEM [9] or Micromegas [10] equipped chambers, are significantly smaller than in wire chambers. We are setting up an experimental ageing measurement. Given the high SLHC rate, we expect that it is not possible to accelerate the measurement by more than a factor 3 .

Efficiency: The efficiency is determined by the probability to create at least one cluster, the distribution of the number of electrons within a cluster, and the efficiency to detect a single electron. In a $1 \mathrm{~mm}$ thick layer of Ar gas (at atmospheric pressure), we expect an efficiency of $95 \%$. With an added quencher gas (Isobutane, n-pentane), efficiencies of $99 \%$ should be within range.

Position resolution: Without diffusion effects, the position resolution in both directions in the CMOS plane is determined by the InGrid hole pitch only. Electrons with the longest possible drift path (and drift time) are subject to diffusion $(\sigma=220 \mu / \sqrt{\mathrm{cm}})$; their weight in the track fit can be adjusted accordingly. In the drift direction, a position resolution of $0.1 \mathrm{~mm}$ is achieved assuming a time resolution of $1.6 \mathrm{~ns}$ and a maximum drift time of $16 \mathrm{~ns}$. The value of the maximum drift time guarantees the determination of the correct SLHC bunch ID.

Rate effects: The ions, created in the avalanche close to the anode pads, cross the amplification gap in $10-20 \mathrm{~ns}$. This means that these ions are almost all vanished before a next bunch crossing occurs. As a result, the amplitude of the avalanches does not depend on the counting rate, as is the case in classical wire chambers. A frac- 
tion of some $1 \%$ of the ions does not end, however, on InGrid. These ions enter the drift gap, and it takes $50 \mu$ s for an ion to travel towards the cathode. A fraction of the ions, created in 2000 bunch crossings, fill the drift gap with a rather homogeneous positive space charge. This space charge causes a variation in the drift velocity and in the drift angle. The angle (which appears as a variation of the Lorentz angle) is maximal at the outer edges of the detector. Both the drift velocity and the local 'Lorentz' angles can be monitored and corrected for.

Radiation hardness: Since the gas will be flushed, the radiation hardness of the detection medium is of no relevance. The radiation hardness of the CMOS chip, made in 130 or $90 \mathrm{~nm}$ technology, is supposed to be sufficient. In the design, care has to be taken that critical Single Event Upsets do not occur.

Power dissipation: Due to the very low anode pad capacity (20 fF), a low-noise and fast (4 ns rise time) preamp dissipates as low as 4 $\mu \mathrm{W}$. Per pixel, some more (digital) circuits are required for the drift time and 'time over threshold' measurements, as well as communication logic. We plan to limit the power dissipation to $0.2 \mathrm{~W} / \mathrm{cm}^{2}$, since this heat can be drained by the gas flow.

Material budget: The CMOS chips can be slimmed down to $30 \mu \mathrm{m}$. In addition, the grid and cathode planes form an equivalent layer of $1 \mu \mathrm{m}$ of aluminium. The equivalent amount of plastic insulating material is less than $1 \mu \mathrm{m}$, and the mass of a layer of $1 \mathrm{~mm}$ Ar gas is negligible.

\section{Conclusions and Outlook}

We have demonstrated that single electrons can be detected with an assembly of a CMOS pixel chip and a Micromegas foil, with an efficiency larger than $90 \%$, in a He based gas mixture. Bubble chamber-like images of cosmic ray tracks have been obtained and even $\delta$-electrons could be observed.

Applied with a thin drift space of one $\mathrm{mm}$, the device could be used as a fast vertex detector in high radiation environments. We are considering to construct a GOSSIP prototype using existing CMOS front-end readout pixels chips like the PSI46 (CMS) or FE (ATLAS).

\section{Acknowledgements}

We thank the Medipix Collaboration for providing us with several wafers with Medipix2 chips, for the readout software and hardware. We would like to thank Wim Gotink and Joop Rövekamp for their creative and essential contributions to the realisation of the test detectors.

\section{REFERENCES}

1. P. Colas, A.P. Colijn, A. Fornaini, Y. Giomataris, H. van der Graaf, E.H.M Heijne, X. Llopart, J. Schmitz, J. Timmermans and J.L. Visschers, Nucl. Instr. and Methods A 535 (2004) 506-510

2. M. Campbell et al., The detection of single electrons by means of a Micromegascovered MediPix2 pixel CMOS readout circuit, Accepted by Nucl. Instr. and Methods A, http://www.arxiv.org/physics/0409048

3. X. Llopart et al., IEEE Trans. Nucl. Sci. 49 (2002) 2279-2283

4. M. Conti et al., IEEE Trans. Nucl Sci. 50 (2003) 869-877

5. D. San Segundo Bello, M. van Beuzekom, P. Jansweijer, H. Verkooijen and J.L. Visschers, Nucl. Instr. and Methods A 509 (2003) 164170

6. Mesa+ Research Institute, University of Twente, The Netherlands.

7. Y. Giomataris et al., Nucl. Instr. and Methods A 376 (1996) 29-35

8. The Micromegas has been made by the CERN EST Workshop.

9. M.C. Altunbas et al., Nucl. Instr. and Meth. A 515 (2003) 249-254

10. G. Puill et al., IEEE Trans. Nucl. Sci 46 (6) (1999) 1894-1897

P. Colas et al., Nucl. Instr. and Meth. A 535 (2004) 181-185 